\section{Endosonographisches Staging des Brochialkarzinoms}

Mit einem Anteil von ca. 20\% aller Todesfälle, die durch Krebserkrankungen verursacht sind - Tendenz steigend stellt das Bronchialkarzinom unverändert eine komplexe interdisziplinäre Herausforderung in der Medizin dar. Prognostisch entscheidend sind zum Zeitpunkt der Erstmanifestation der Zelltyp sowie das Tumorstadium. Aus diesem Grund sehen die Empfehlungen zur Diagnostik des Bronchialkarzinoms ein Standardprogramm vor, das neben der feingeweblichen Sicherung des Primärtumors eine ausgedehnte Suche nach Metastasen vorsieht, wobei ein besonderes Gewicht auf die präzise Analyse der mediastinalen Lymphknotenbeteiligung insbesondere bei Patienten mit nicht kleinzelligem Bronchialkarzinom gelegt wird $[1,2]$. Da die Operation des nicht kleinzelligen Bronchialkarzinoms zum gegenwärtigen Zeitpunkt integraler Bestandteil einer kurativen Behandlung ist, kommt der exakten Beurteilung einer Lymphknotenbeteiligung des Mediastinums eine herausragende Bedeutung zu, um eine individuelle Risikoabschätzung und optimale Therapieplanung machen zu können. Die derzeitige Diskussion über die Möglichkeiten eines neoadjuvanten Therapiekonzeptes unter Vorschaltung einer Chemo-(Radio-)Therapie bei den Tumorstadien, die bereits eine Beteiligung mediastinaler Lymphknoten aufweisen (Stadium IIIA/IIIB)m unterstreicht eindrucksvoll diese Aussage $[3,4]$.

In den vergangenen Jahren haben einige neue diagnostische Verfahren Aufmerksamkeit hervorgerufen, die möglicherweise die Chance für ein exakteres Tumor-Staging und damit für eine verbesserte Therapieplanung bieten können. An dieser Stelle seien die Magnetresonanztomographie (MR), die Positronen-Emissions-Tomographie (PET) [5] sowie die endoösophageale und endobronchiale Sonographie des Mediastinums (EUS bzw. EBUS) [6,7] genannt. Die Hoffnung, die sich an diese Verfahren knüpft, ist um so größer, als die Daten der letzten Jahre zeigen konnten, dass es innerhalb des quantitativ mit 75\% gewichtigen Anteils der nicht kleinzelligen Bronchialkarzinome $\mathrm{zu}$ einer bisher noch nicht geklärten Abnahme des Plattenepithelkarzinoms bei gleichzeitiger $\mathrm{Zu}$ nahme des Adenokarzinoms gekommen ist, wobei gerade diese Tumorform sich durch eine frühe ausgedehnte Metastasierung auszeichnet [8].

Im Folgenden soll nun im Wesentlichen auf die Möglichkeit der Endosonographie für die Beurteilung des Mediastinums

Pneumologie 2000; 54: 539-541

(c) Georg Thieme Verlag Stuttgart · New York ISSN 0934-8387

\begin{abstract}
A. Pforte
Medizinische Kernklinik und Poliklinik, Bereich Pneumologie, Universitäts-Krankenhaus Eppendorf, Hamburg
\end{abstract}

eingegangen werden. Mit dieser Methode besteht in den Händen von Gastroenterologen bereits eine langjährige Erfahrung im Hinblick auf die Diagnostik von Tumoren des oberen Gastrointestinaltraktes. Endosonographische Aussagen zur Ausdehnung und Lokalisation des Primärtumors sowie zum Lymphknotenstatus spielen für die Beurteilung der Operabilität inzwischen eine große Rolle.

Mit der endoösophagealen Sonographie, die mit einem Seitblick-Endoskop und aufgesetzter Ultraschall-Einheit erfolgt, ist die Beurteilung von Strukturen ab einer Größe von ca. $3 \mathrm{~mm}$ im Umkreis von $5 \mathrm{~cm}$ des Ösophagus möglich. Neben dem herkömmlichen Radialschallkopf, der ein $360^{\circ}$-Bild senkrecht zur Geräteachse erzeugt, kommt zunehmend auch ein linearer Sektor-Scanner zur Anwendung, der einen $110^{\circ}$-Bildschnitt parallel zur Geräteachse erlaubt. Auf diese Weise kann das gesamte posteriore Mediastinum eingesehen werden, das aortopulmonale Fenster und die Umgebung der großen Gefäße. Eine entscheidende diagnostische Erweiterung erfuhr die Methode durch die Kombination des Schallkopfes mit einer Punktionseinheit, die die Durchführung einer Feinnadelbiopsie im Rahmen der Endosonographie ermöglicht $[9,10]$. Rasch ergab sich eine klinisch interessante Erweiterung der Indikationsstellung der EUS auf solide Tumoren des Mediastinums und in einem weiteren Schritt auf die Evaluation der Lymphknotenbeteiligung beim Bronchialkarzinom [11,12]. In den vergangenen Jahren hat eine Reihe von Arbeitsgruppen über erste Erfahrungen mit dieser Methode berichtet, in denen sehr eindrucksvoll gezeigt werden konnte, dass es mit der endosonographisch gesteuerten Feinnadelpunktion möglich ist, mediastinale Raumforderungen darzustellen und durch die Punktion deren Dignität zu klären.

1982 veröffentlichten Silvestri et al. eine Studie, in der erstmals die endoösophageale Sonographie mit Feinnadelpunktion und das Thorax-CT in einer vergleichenden Untersuchung gegenübergestellt wurden. Dabei ergaben sich eine Sensitivität und Treffsicherheit von 89 bzw. 100\% für die EUS verglichen mit 82 bzw. 38\% für das CT [13]. In weiteren Studien von Giovannini et al. sowie Gress et al. ließen die Daten noch deutlicher die Überlegenheit der Endosonographie gegenüber dem CT erkennen $[11,14]$. Ein wesentlicher Grund hierfür liegt darin, dass beim CT das entscheidende Kriterium für die Malignität einer Raumforderung die Größe darstellt: Erst ab einem Durchmesser $>1 \mathrm{~cm}$ in der kurzen Achse wird ein Lymphknoten als pathologisch beurteilt. Anders verhält es sich bei der sonographischen Beurteilung, bei der andere morphologische Merkmale wie die Unterscheidung in echoarme und echoreiche Strukturen sowie der 
Nachweis von kleinen Gefäßformationen innerhalb der Raumforderung von diagnostischer Bedeutung sind.

Nun ist durch chirurgische Studien anhand von Thorakotomien mit radikaler Lymphadenektomie eindrucksvoll belegt, dass ein metastatischer Befall bereits in Lymphknoten von einer Größe $<1 \mathrm{~cm}$ vorliegen kann. Berücksichtigt man die Ergebnisse der vorliegenden Studien, ergibt sich für das CT im Hinblick auf die Beteiligung mediastinaler Lymphknoten eine Sensitivität, die zwischen 29 und $90 \%$, sowie eine Spezifität, die zwischen 54 und $93 \%$ schwanken kann $[15,16]$.

Ein weiterer wichtiger Aspekt betrifft das bisher nicht einheitliche chirurgische Vorgehen zur Lymphknotendokumentation und Lymphadenektomie beim Bronchialkarzinom. Einer Umfrage von B. Passlick und O. Thetter zufolge wird die Entnahme mediastinaler Lymphknoten in 59\% der beteiligten thoraxchirurgischen Abteilungen unter Berücksichtigung des intraoperativen makroskopischen Aspekts durchgeführt, während in $41 \%$ der Abteilungen eine systematische En-blockResektion erfolgt [17].

Berücksichtigt man die Tatsache, dass bei $50 \%$ der Patienten, die im Stadium 1 bis IIIA operiert werden, postoperativ eine erneute Tumormanifestation auftritt, die einen wesentlichen verantwortlichen Faktor für die hohe Mortalität der Erkrankung darstellt, so muss man sich fragen, inwieweit ein metastatischer Befall mediastinaler Lymphknoten vorgelegen haben kann, der weder präoperativ noch intraoperativ gesichert wurde, so dass nicht die entsprechenden Weichen für eine weiterführende Therapie gestellt wurden.

Hier kommt ein grosser Vorteil der Endosonographie gegenüber der Computertomographie in der präoperativen Beurteilung zum Tragen. Den entscheidenden diagnostischen Schritt stellt die im Rahmen der Untersuchung durchgeführte Feinnadelpunktion der sonographisch dargestellten Lymphknoten dar, die auch bei Strukturen von einer Größe $<1 \mathrm{~cm}$ ohne technische Probleme möglich ist und eine zytologisches Verifizierung des bildgebenden Befundes ermöglicht.

Als leitlinienorientiertes Vorgehen wird trotz der aufgezeigten Probleme bisher das Thorax-CT als Standarduntersuchung zum Staging des Mediastinums [2] empfohlen. Dabei wird Bezug genommen auf Studien, die zeigen konnten, dass das CT ebenso wie die Mediastinoskopie - teilweise sogar besser - in der Lage ist, überflüssige Thorakotomien zu vermeiden. Weitere Gründe für eine Zurückhaltung gegenüber der Mediastinoskopie liegen in ihrer obligaten Durchführung in Allgemeinanästhesie und unter stationären Bedingungen sowie die nicht unbeträchtliche Komplikationsrate von 3-4\% [18]. Die derzeitigen Empfehlungen der DGP betonen allerdings die Bedeutung der Mediastinoskopie bei linksseitigen Bronchialkarzinomen wegen der häufigeren Metastasierung auf die kontralaterale Seite [1].

Zur Standortbestimmung der Rolle der endoösophagealen Sonographie als Routineuntersuchung im Rahmen des mediastinalen Stagings des Bronchialkarzinoms wäre eine kontrollierte Studie wünschenswert, in der die Mediastinoskopie als „Goldstandard“ mit der Endosonographie direkt verglichen und eine Bestätigung durch die intraoperative Klärung des Befundes herbeigeführt würde. Hierbei muss man sich aber darüber im klaren sein, dass die Aussagekraft einer solchen Untersuchung dadurch erheblich eingeschränkt wäre, dass Endosonographie und Mediastinoskopie nicht in der Lage sind, identische Lymphknotenstationen zu erfassen. Der Zugang der zervikalen bzw. anterioren Mediastinoskopie erlaubt eine exzellente Beurteilung des vorderen Mediastinums mit prä- und paratrachealen Lymphknoten. Die Endosonographie, ausgehend vom Ösophagus, gestattet die Erfassung der Lymphknotenstationen des hinteren Mediastinums. Beide Methoden erreichen allerdings gemeinsam das aortopulmonale Fenster. Somit müssen die beiden Verfahren als ergänzende Untersuchungsmethoden betrachtet werden, die individuell allein oder in Kombination zum Einsatz kommen können. Verwiesen werden soll an dieser Stelle auf die bisher einzige Untersuchung zu dieser Fragestellung, die trotz dieser grundsätzlichen Einwände anhand der vergleichenden Anwendung von EUS und Mediastinoskopie an 21 Patienten zeigen konnte, dass mit beiden Methoden vergleichbare Resultate in der Diagnostik tumorös befallener mediastinaler Lymphknoten zu erzielen waren [19].

Insgesamt lässt sich eine Reihe von schwerwiegenden Argumenten zusammentragen, die für eine großzügige Anwendung der Endosonographie mit Feinnadelpunktion im Rahmen des Stagings des Bronchialkarzinoms sprechen und die der Methode einen Platz in der zeitlichen Abfolge des Untersuchungsganges vor einer Mediastinoskopie einräumen. Zum einen sei hier die Möglichkeit der ambulanten Durchführung genannt und zum andern das Fehlen von Mitteilungen über ernsthafte Komplikationen dieser Untersuchung in der Literatur, was anhand des eigenen Patientenkollektivs bestätigt werden kann. Weiterhin ist die Möglichkeit der wenig invasiven Sicherung der zytologischen/histologischen Diagnose durch die EUS hoch zu bewerten, wenn diese auf Grund der Lokalisation des Primärtumors durch bronchoskopische Verfahren bzw. transthorakale Biopsien nicht möglich ist, wovon in $10-20 \%$ der Fälle auszugehen ist [20].

Ein weiteres lndikationsgebiet stellt die Differenzialdiagnose zu entzündlichen Erkrankungen im Mediastinum, insbesondere der Lymphknotenvergrößerung im Rahmen der Sarkoidose dar. Auch für diese Diagnose konnte eine hohe Treffsicherheit der EUS mit Feinnadelpunktion bestätigt werden [21]. Sollte die EUS keine ausreichende Klärung der jeweiligen Fragestellung ermöglichen, so ist im Einzelfall abzuwägen, ob zunächst die Mediastinoskopie oder auf direktem Wege die Thorakotomie anzustreben ist.

Ein weiterer interessanter Aspekt betrifft die Möglichkeit des endobronchialen Ultraschall (EBUS), der hinsichtlich Untersuchungstechnik und Interpretation besondere Anforderungen stellt, da entweder Artefakte der Bildgebung durch Luftüberlagerung oder eine kurzfristige Ballonblockade von Trachea oder großen Bronchien für die Durchführung der Untersuchung in Kauf genommen werden müssen. Die bisher durchgeführten Studien zeigen, dass hiläre und peribronchiale Lymphknoten exakt dargestellt werden können ebenso wie pulmonale Gefäße, so dass durch dieses Vorgehen die Planung transbronchialer und transtrachealer Feinnadelpunktionen optimiert werden kann $[22,23]$. Ein weiteres Anwendungsgebiet ist die Darstellung peripherer pulmonaler Herde sowie die Tiefendiagnostik einer Tumorausdehnung innerhalb der Bronchialwand. 
Im Hinblick auf innovative Verfahren wie die verbesserte lokale Diagnostik von Frühmanifestationen eines Bronchialkarzinoms i. S. eines Carcinoma in situ und Therapie mittels Fluoreszenzbronchoskopie dürfte die EBUS ein großes Potenzial für die exakte Therapieplanung und Verlaufskontrolle bieten, wobei zum derzeitigen Zeitpunkt nur über klinische Studien mit relativ niedrigen Fallzahlen diskutiert werden kann. Die Endosonographie beinhaltet somit insgesamt eine Reihe von Eigenschaften, die dieser Methode einen hohen Rang im Rahmen des Staging des Bronchialkarzinoms zubilligen. Von entscheidender Bedeutung ist aus meiner Sicht die Kombination von Bildgebung und Biopsie-Möglichkeit bei gleichzeitig geringer Belastung für den Patienten. Ebenfalls beeindruckend sind das Risikoprofil und die niedrigen Kosten dieses Verfahrens [24].

Der Endosonographie ist eine weite Verbreitung gerade bei Pneumologen zu wünschen, die sich dieser Methode ebenso wie auch der transthorakalen Sonographie verstärkt widmen sollten, da die einfache Durchführbarkeit und das Fehlen einer Strahlenbelastung im Einzelfall von großer Bedeutung sein können. Durch die Etablierung der Methode an weiteren Zentren wird es möglich sein, in Zukunft Studien mit größeren Patientenkollektiven durchzuführen, denn nur hierdurch ist eine objektive Evaluierung dieser Methode möglich. Wichtige offene Fragen betreffen die Standardisierung der Lymphknotenpunktion sowie die Rolle der Endosonographie in der Verlaufskontrolle des Bronchialkarzinoms.

\section{Literatur}

${ }^{1}$ Deutsche Gesellschaft für Pneumologle und Deutsche Gesellschaft für Thorax-, Herz- und Gefäßchirurgie. Empfehlungen zur Diagnostik, Stadieneinteilung und operativen Therapie des Bronchialkarzinoms. Pneumologie 1994; 48: 262 - 269

${ }^{2}$ American Thoracic Society/European Respiratory Society. Pretreatment Evaluation of Non-Small-Cell Lung Cancer. Am J Respir Crit Care Med 1997; 156: 320-332

${ }^{3}$ Einhorn LH. Neoadjuvant and Adjuvant Trials in Non-Small Cell Lung Cancer. Ann Thorac Surg 1998; 65: 208 -211

${ }^{4}$ Thomas M, Macha HN, Rübe Oh, van de Loo J. Stellenwert der Chemotherapie in der multimodalen Behandlung des nicht kleinzelligen Bronchialkarzinoms. DMW 1995; 120: 1627-1630

${ }^{5}$ Hoh CK, Schiepers C, Seltzer MA, Gambhir SS, Silverman DHS, Ozernin J, Maddahi J, Phelps ME. PET in Oncology: Will it Replace the Other Modalities? Seminars in Nuclear Medicine 1997; XXVII, 2: 94-106

${ }^{6}$ Grimm H, Binmoeller KF, Hamper K et al. Endosonography for preoperative locoregional staging of esophageal and gastric cancer. Endoscopy 1993; 25: $224-230$

${ }^{7}$ Becker H. Endobronchialer Ultraschall - Eine neue Perspektive in der Bronchologie. Ultraschall in Med 1996; 17: 106 -112

${ }^{8}$ Hoffman PC, Mauer AM, Vokes EE. Lung cancer. Lancet 2000; 355: $479-485$

${ }^{9}$ Pedersen BH, Vilmann P, Folke K, Jacobsen GK, Krasnik M, Milman N, Hancke S. Endoscopic Ultrasonography and Real-time Guided Fine-needle Aspiration - Biopsy of Solid Lesions of the Mediastinum Suspected of Malignancy. Chest 1996; 110, 2: $539-544$

${ }^{10}$ Vilman P, Hancke S, Henriksen FW, Jacobsen GK. Endosonographically-Guided Fine Needle Aspiration Biopsy of Malignant Lesions in the Upper Gastroentestinal Tract. Endoscopy 1993; 25: $523-527$
${ }^{11}$ Gress FG, Savides TJ, Sandler A, Kesler K, Conces D, Cummings O, Mathur P, lkenberry 5, Bilderback 5, Hawes R. Endoscopic Ultrasonography, Fine-Needle Aspiration Biopsy Guided by Endoscopic Ultrasonography, and Computed Tomography in the Preoperative Staging of Non-Small-Cell Lung Cancer: A Comparison Study. Annals of Internal Medicine 1997; 127: 604-612

${ }^{12}$ Hawes RH, Gress F, Kesler K, Cummings OW, Concec DH. Endoscopic ultrasound versus computed tomography in the evaluation of the mediastinum in patients with non small celI lung cancer. Endoscopy 1994; 26: $784-787$

${ }^{13}$ Silvestri GA, Hoffman BJ, Bhutani M5, Hawes RH, Coppage L, Sanders-Cliette A, Reed CE. Endoscopic Ultrasound With FineNeedle Aspiration in the Diagnosis and Staging of Lung Cancer. Ann Thorac Surg 1996; 61: 1441 - 1446

${ }^{14}$ Giovannini M, Seitz J-F, Monges G, Perrier H, Rabbia 1. Fineneedle aspiration cytology guided by endoscopic ultrasonography: Results in 141 patient. Endoscopy 1995; 27: 171-177

${ }^{15}$ Arita T, Kuramitsu T, Kawamura M, Matsumoto T, Matsunaga N, Sugi K, Esato K. Bronchogenic carcinoma: incidence of metastases of normal sized lymph nodes. Thorax 1995; 5: 1267-1269

${ }^{16}$ lzbicki JR, Thetter O, Karg O, Kreusser T, Passlick B, Trupka A, Häußinger K, Woeckel W, Kenn RW, Wilker D, Limmer J, Schweiberer L. Accuracy of computed tomographic scan and surgical assessment for staging of bronchial carcinoma. The Journal of Thoracic and Cardiovascular Surgery 1991; 104: 413

17 Passlick B, Thetter O. Lymphknotendokumentation und Lymphadenektomie bei Bronchialcarcinomen. Der Chirurg 1997; 68: $601-605$

${ }^{18}$ Kelm C, Henneking K, Zimmermann T, Padberg W. Die Bedeutung der Mediastinoskopie für das korrekte Lymphknotenstaging. Zentralblatt für Chirurgie 1996; 121: 84-86

${ }^{19}$ Serna DL, Aryan HE, Chang KJ, Brenner M, Tran LM, Chen JC. An Early Comparison between Endoscopic Ultrasound-guided Fineneedle Aspiration and Mediastinoscopy for Diagnosis of Mediastinal Malignancy. The American Surgeon 1998; 64: $1014-1018$

${ }^{20}$ Fritscher-Ravens A, Soehendra N, Schirrow L, Sriram PVJ, Meyer A, Hauber H-P, Pforte A. Role of Transesophageal Endosonography Guided Fine-Needle Aspiration in the Diagnosis of Lung Cancer. Chest 2000; 117: 339-345

${ }^{21}$ Fritscher-Ravens A, Parupudi VJS, Topalidis T, Hauber HP, Meyer A, Soehendra N, Pforte A. Diagnosing Sarccoidosis Using Endosonography-Guided Fine-Needle Aspiration. Chest 2000, in press

${ }^{22}$ Shannon JJ, Bude RO, Orens JB, Becker FS, Whyte Rl, Rubin JM, Quint LE, Martinez FJ. Endobronchial Ultrasound-Guided Needle Aspiration of Mediastinal Adenopathy. Am J Respir Crt Care Med 1996; 153: $1424-1430$

${ }^{23}$ Kurimoto N, Murayama M, Yoshioka S, Nishisaka T, Inai K, Dohi K. Assessment of Usefulness of Endobronchial Ultrasonography in Determination of Depth of Tracheobronchial Tumor Invasion. Chest 1999; 115: 1500-1506

${ }^{24}$ Fritscher-Ravens A, Pforte A. Endosonographisch gesteuerte Feinnadelaspirationsbiopsie - Stellenwert in Diagnostik und Staging des Bronchialkarzinoms. Deutsches Ärzteblatt, 1999; 96: $2982-2986$

Prof. Dr. med. Almuth Pforte

Universitäts-Krankenhaus Eppendorf Medizinische Kernklinik und Poliklinik Bereich Pneumologie

Martinistraße 52

20246 Hamburg 